AperTO - Archivio Istituzionale Open Access dell'Università di Torino

\title{
A competitive idea-based growth model
}

\section{This is the author's manuscript}

Original Citation:

Availability:

This version is available http://hdl.handle.net/2318/1702121

since 2020-03-10T13:05:05Z

Published version:

DOI:10.1080/10438599.2019.1616663

Terms of use:

Open Access

Anyone can freely access the full text of works made available as "Open Access". Works made available under a Creative Commons license can be used according to the terms and conditions of said license. Use of all other works requires consent of the right holder (author or publisher) if not exempted from copyright protection by the applicable law. 


\title{
A Competitive Idea-Based Growth Model
}

\author{
Carla Marchese $^{\mathrm{a}}$ and Fabio Privileggi ${ }^{\mathrm{b}}$ \\ anstitute POLIS - DiGSPES, Università del Piemonte Orientale "Amedeo Avogadro", Via \\ Cavour 84, 15121 Alessandria (Italy). Email: carla.marchese@uniupo.it, ORCID \\ 0000-0001-9935-2038; bDept. of Economics and Statistics "Cognetti de Martiis", Università \\ di Torino, Lungo Dora Siena 100 A, 10153 Torino (Italy), ORCID 0000-0002-8837-2006
}

\begin{abstract}
ARTICLE HISTORY
Compiled April 29, 2019

ABSTRACT

In this paper we present a model in which endogenous growth arises in competitive markets. Knowledge is described as a factor used directly in the final goods' production. Firms demand both basic nonrival knowledge contents, which are supplied jointly and inelastically with raw labor, and further contents supplied by patent holders. This fact, together with Lindahl prices for knowledge, allows competition to work, while it also implies that workers' income share declines overtime. In a first version of the model with constant cost of knowledge production the first best is attained. In a further version of the model, in which the cost of knowledge production is allowed to change over time and thus intertemporal externalities arise, in a decentralized economy a second best equilibrium occurs in the transitional period, while in the long run there is convergence to efficiency.
\end{abstract}

\section{JEL CLASSIFICATION}

C61; E10; O31; O41

\section{KEYWORDS}

Endogenous growth; Competitive markets; Intangible inputs; Lindahl prices

'Socrates: There are beds and tables in the world-plenty of them, are there not? Glaucon: Yes.

Socrates: But there are only two ideas or forms of them-one the idea of a bed, the other of a table.

—Plato, The Republic, Book X, dialogue between Socrates and Glaucon, 380 BC'

\section{Introduction}

The motivation for this paper is to further pursue the challenging notion of 'competitive innovation', in a framework in which, however, the classical view about the nonrival nature of knowledge is maintained. In fact, we aim at providing a parsimonious set of assumptions sufficient at supporting the viability of competition in the whole economy, that is, price taking behavior and zero profits at all levels, as well as market incentives for R\&D activities and thus sustained economic growth. While the 
assumptions we arrive at are demanding, they capture some features that might prevail in the economy thanks to the characteristics that technological change assumed in the last decades. The first feature we consider is that technical progress leads more and more to the provision of information goods or knowledge products (Scotchmer 2005) such as computer programs, internet applications, business models, etc., which are patentable ${ }^{1}$ and directly usable in the final goods production. It thus seems appropriate to assume that knowledge can also enter directly into the final goods production function, without having to be incorporated into intermediate goods or into labor (as, e.g., in human capital models). ${ }^{2}$ This direct penetration of immaterial contents into final goods production also implies that much more information than in the past is available to asses the value of the marginal product of knowledge (Gray and Grimaud 2016).

The second feature that we consider is the "routinization" process (Acemoglu and Autor 2011), which implies the possibility of codifying and automating many tasksincluding cognitive ones - previously performed by workers. Bringing this trend to its limit, this substitution process in the medium-upper tier of the labor market would spare only creative tasks, which imply the elaboration of new models and ideas. But then the suppliers of such ideas need no more to be employees. ${ }^{3}$ Entrepreneurs who operate under the protection of laws on Intellectual Property Rights (IPR) are well suited to supply knowledge contents. One can thus expect that patented knowledge has the potential for widely displacing both medium and high-skilled workers. While on the other hand robotization has in principle the potential for substituting also the remaining tasks, i.e., those of low-skilled workers, the perspective diffusion and economic impact of robots is still questioned (Gordon 2014). The problem is mainly represented by the difficulty of routinization of tasks which require a level of adaptability and responsiveness which is nowadays difficult to achieve. Hence, at least for a while the process of substitution should spare low-skilled workers, endowed with the basic levels of knowledge, which are still needed to exploit patented intellectual contents. ${ }^{4}$

In this paper, to describe the final goods' production, we use a general neoclassical production function $Y=F(X, A L)$-where $Y$ is a composite consumption good, $A$ stands for knowledge, $L$ for labor and $X$ for an intermediate good - exhibiting constant returns to scale in two variables, $X$ and the product $(A L)$. We assume that workers inelastically supply basic knowledge, representing their inherited common cultural endowment, jointly with raw labor, while knowledge advances are supplied only by patent holders. Since intellectual contents coming from these two sources are perfect substitutes when used in the production of final goods, they are treated as just one factor, i.e., knowledge, a nonrival but excludable good. Since workers supply of raw labor and basic knowledge are physically joint and inelastic, they are willing to supply both factors provided that at least one of them has a positive price. Firms producing final goods demand just knowledge from both types of providers, while raw labor

\footnotetext{
${ }^{1}$ This trend for the U.S. can be dated back to 1998, when in the so called State Street Bank case a business method was declared patentable. Many other similar rulings followed with respect to software. For patentability in general see Eckert and Langinier (2013).

${ }^{2}$ For the case in which labor and individual licences for accessing disembodied knowledge are jointly demanded, see Marchese and Privileggi (2018).

${ }^{3}$ We envisage a potential turning point and thus depart from Acemoglu and Restrepo (2016) who maintain that new and more complex tasks for labor can always be created.

${ }^{4}$ Arrieta Ibarra et al. (2017) stress also the role of data produced by human interactions in the generation of information useful for Machine Learning. They envisage a world of "Data as Labor", where workers are identified with the suppliers of such information, i.e., of basic intangible intellectual contents, and paid accordingly.
} 
represents an externality of basic knowledge.

A technical ingredient that renders competition viable and efficiently allocates workers to the single firms, internalizing the externality, is represented by the Lindahl prices paid by the final goods' sector to compensate the suppliers of knowledge. As will become more clear in Section 3.4, the larger the firm's dimension in terms of output (and thus the larger the number of workers it hires), the larger its revealed demand price for knowledge (since raw labor acts as a multiplier of the marginal product of knowledge); but a larger Lindahl price implies also a larger firm's payment for the workers' basic knowledge, or, so to say, a larger "payroll". Hence, Lindahl prices support the exact equivalent of a competitive labor market equilibrium.

The key idea about the viability of competition in the R\&D market may be summarized as follows. Final good producers (in large number), besides the intermediate good $X$ demand only knowledge $A$ and are eager to pay the Lindahl price; patent holders (in large number) are willing to rent the positive stock of knowledge they already own whenever its price is positive. The price arises from the intersection between the (strictly decreasing) demand for knowledge with knowledge supply, where the latter is fixed at each instant because it originates from the available stock of already patented inventions, whose services, unlike in a Dixit-Stiglitz framework, are assumed to be perfect substitutes when used in production. Future Lindahl prices, when discounted at any previous instant according to the market interest rate, turn out to cover the cost of producing new inventions by R\&D firms, which are thus incentivized to undertake research activities and eventually allow sustained endogenous growth of the economy. As R\&D activities occur under a free entry condition, R\&D firms earn zero profits and are thus competitive like any other actor in the economy, specifically, the firms in the final good sector.

While in the basic version of the model knowledge used in production is fully excludable, so that the first best is reached, in a further version externalities arise and a second best result is attained in the transitional period. Even in the latter case, however, in the long run the decentralized solution converges to the optimal Asymptotic Balanced Growth Path (ABGP).

Our results contribute to the debate about the motivations of the decline of the income share of labor at the advantage of the share going to intangibles and particularly to holders of patents, a stylized fact that has attracted much attention (Corrado, Hulten, and Sichel 2009; Karabarbounis and Neiman 2014; Koh, Santaeulàlia-Llopis, and Zheng 2016). A novel contribution of the paper is actually that of providing a rationale for a declining workers' income share in a growing competitive economy. This result arises because, as economic growth proceeds, the share of knowledge owned by patent holders increases, as knowledge grows only thanks to research protected by IPRs.

The paper is organized as follows: after a discussion of the related literature in Paragraph 2, in Section 3 the basic model is presented and its static equilibrium is thoroughly described, while Section 4 is devoted to the ensuing dynamic equilibrium. In Section 5 we consider the case in which the cost of knowledge production is not constant overtime, establishing that the decentralized equilibrium attains Pareto optimality only in the long-run. Finally, Section 6 reports some conclusions. All mathematical proofs are postponed in the Appendix.

\section{Discussion on Related Literature}

Hellwig and Irmen (2001) and Boldrin and Levine (2008) are among the notable excep- 
tions to the mainstream paradigm in the endogenous growth literature, which maintains that a non-competitive market must occur somewhere in the economy, in order to provide private economic incentives for research. ${ }^{5}$ Hellwig and Irmen (2001) show that atomless competitive markets in the production sector can finance the fixed cost of research out of inframarginal rents, an opportunity allowed by strictly increasing marginal costs at the level of each individual firm. In their model, the returns from innovation are privately appropriable only in the introductory period. Information about new findings spills over afterward, thus paving the way for further innovation in all the firms, with increasing returns at the aggregate level and economic growth. The presence of externalities implies, however, that the first best is not achieved. Boldrin and Levine (2008), instead, depart from the assumption of ideas as nonrival goods by noting that the means of access to knowledge (such as the template of a new good) are rival and excludable. Moreover, replicating the first copy is time-consuming, and this fact, coupled with the impatience of consumers, implies that in a competitive market a scarcity rent will be paid by buyers. The inventor can thus sell the first copy at a price - which depends on the value of the services it delivers in terms of consumption and of replicability into further copies - earning a rent that may be sufficiently large to finance the indivisible cost born to produce the innovation. Whenever research activity can be financed this way, economic growth becomes viable even if the inventor is not granted a patent and thus has no IPR over further copies.

Our contribution exploits some hints from the Boldrin and Levine approach, while developing them into a more standard endogenous growth model, in which knowledge is described as a continuous excludable public good. In fact, even if Boldrin and Levine criticize the common tenet on the public good nature of knowledge, one might rationalize as Lindahl prices the rents they consider, as the latter derive from multiple uses of one initial invention. However, Lindahl prices in the Boldrin and Levine model are paid jointly with the cost of each copy, i.e., a private rival good, while we consider disembodied knowledge instead. Equilibria based on Lindahl prices are often deemed unrealistic because of the problem of "revelation" of demand. Boldrin and Levine do not systematically tackle this problem: they just quote examples supporting the idea that in general rents will be large enough to finance most useful innovations. We explicitly tackle the problem of demand revelation.

As for knowledge use, following an approach similar to ours, also Chantrel, Grimaud, and Tournemaine (2012) assume that disembodied knowledge is being directly used in the production of final goods. Although starting from different assumptions, they show as well that competition in the market for knowledge is sustainable under perfect excludability and with Lindahl prices, so that the distortion caused by knowledge spillovers vanishes. However, Chantrel, Grimaud, and Tournemaine (2012) assume that the final goods' production occurs under increasing returns, so that competition is not viable in that market.

\section{Constant Cost of Knowledge Production}

Our model is a Ramsey-type model of growth with endogenous creation of knowledge. The economy is composed of households, firms and the government. Households receive

\footnotetext{
${ }^{5}$ The idea that market power is necessary for inducing costly research dates back to Schumpeter (1911). It was brought up again in the '90s by many papers (besides Romer 1990, see, e.g., also Grossman and Helpman 1991, Peretto 1996 and Aghion and Howitt 1998) and subsequently it has become a widely shared tenet in the literature on endogenous economic growth.
} 
compensations for supplying inputs to the production sector, purchase a composite consumption good, which also represents the numeraire, and choose how much to save in order to accumulate new knowledge. There are two types of firms: one performing knowledge creation activities ( $R \& D$-firms) and one producing the final consumption good ( $F$-firms).

\subsection{Households}

We shed population growth ${ }^{6}$ and assume that the size of the economy-i.e., the total number of households - is constant. We adopt the standard assumption that all households have the same rate of time preference, $\rho>0$, and an identical increasing and concave instantaneous utility function.

Assumption 3.1. The aggregate representative consumer is endowed with an instantaneous objective $u(C)$, where $C$ is aggregate consumption, with $u^{\prime}(C)>0$ and $u^{\prime \prime}(C)<0$.

Households' goal consists of choosing consumption in order to maximize their own lifetime discounted utility subject to the usual asset accumulation constraint, and their initial common knowledge endowment $A(0)=A_{0}>0$. Knowledge is assumed to be a nonrival good. The initial knowledge endowment $A_{0}$ belongs to the households in the sense that they share it as a part of their cultural heritage. $A_{0}$, however, is excludable whenever its productive uses involving firms are considered, and each household is entitled to an equal share of the compensations the firms must pay for its exploitation. ${ }^{7}$ Households supply $A_{0}$ to the final good producers at all instants $t \geq 0$ jointly with constant raw labor ${ }^{8} L(t) \equiv L$. Thanks to such joint inelastic supply of $L$ and $A_{0}$, workers are willing to provide raw labor even if they are compensated only as owners of their initial knowledge endowment $A_{0}$, whenever such compensation is strictly positive.

As the representative household earns only royalties from renting knowledge to $F$ firms, she faces the following maximization problem,

$$
\begin{gathered}
\max _{[C(t)]_{t=0}^{\infty}} \int_{0}^{+\infty} u[C(t)] e^{-\rho t} \mathrm{dt} \\
\text { subject to } \dot{B}(t)=r(t) B(t)-C(t),
\end{gathered}
$$

where $B(t)$ denotes an asset that will be specified later on and $r(t)$ is the market rate of return on assets, with the additional constraint $0 \leq C(t) \leq r(t) B(t)$, for a given initial asset level $B(0)=B_{0}>0$. Standard analysis of the (concave) current-value Hamiltonian associated to (1) yields the following necessary condition of optimality, which is the well-known Euler equation:

$$
\frac{\dot{C}(t)}{C(t)}=\frac{1}{\varepsilon_{u}[C(t)]}[r(t)-\rho]
$$

where $1 / \varepsilon_{u}(C)=-u^{\prime}(C) /\left[u^{\prime \prime}(C) C\right]$ is the intertemporal elasticity of substitution.

\footnotetext{
${ }^{6}$ For an extension in which population growth is considered see the working paper version of this paper (Marchese and Privileggi 2016).

${ }^{7} \mathrm{By}$ a continuity argument, the knowledge endowment of workers at the initial instant of time provides a marginal benefit and commands a price equal to that of the further contents produced under patents' protection.

${ }^{8}$ For the sake of simplicity it is assumed that labor $L$ is a continuous variable.
} 


\subsection{RED Sector}

Throughout the whole paper we will resort to the standard simplified approach that describes aggregate knowledge creation as a deterministic process. That is, we assume that there is no aggregate uncertainty in the innovation process while, of course, there may be idiosyncratic uncertainty. ${ }^{9}$ In order to obtain an infinitely lived and fully enforced patent, each idea produced by a $R \& D$-firm must be new and differentiated, while, as will be clarified in the next section, when knowledge is used by the $F$-firms it behaves as a homogeneous good.

In this first version of the model we simplify things by considering a constant cost of new knowledge production, an assumption that will be relaxed later on.

Assumption 3.2. Each new idea can be produced at a constant unit cost, $\eta(t) \equiv$ $\eta>0$.

Under Assumption 3.2 the innovation possibilities frontier is given by

$$
\dot{A}=\frac{J}{\eta},
$$

where $J$ represents investment in new knowledge production. We shall assume that there is free entry into R\&D activities, that is, any individual or firm can spend one unit of its wealth at time $t$ to generate a flow rate $1 / \eta$ of new ideas. Every $R \& D$-firm produces new knowledge and aims at profit maximization. Free entry in the business of producing new ideas implies that profits must be zero in equilibrium. Hence, the value of the patent associated to each (differentiated) unit of new knowledge purchased by households at instant $t$ corresponds to the same constant: $\eta>0$.

\subsection{Producing Sector}

In the final good sector $F$-firms are competitive and operate in a standard neoclassical framework: at each instant $t F$-firm $i$ employs a composite intermediate good and knowledge-augmented labor to produce a composite consumption good according to a neoclassical production function, $Y_{i}=F\left(X_{i}, A_{i} L_{i}\right)$. The intermediate good $X$ is made up of final goods destined to production, so that its price is the numeraire, $p^{X}=1$, while $L$ is raw labor provided by a large and constant population and $A$ denotes knowledge. Recall that knowledge is assumed to be nonrival and perfectly excludable. ${ }^{10}$ Specifically, from the $F$-firms perspective $A$ is an aggregate composition of perfect substitutes, implying that whichever new idea is added to the stock, it has the same marginal productivity of all other ideas - including those forming the initially inherited endowment $A_{0}$. Knowledge can be directly used in production, while labor $L$ augments its productivity. Because labor is supplied inelastically and jointly with the initial knowledge $A_{0}$, while all other inputs are for sale, $F$-firms demand only the intermediate goods $X$ and knowledge $A$. Input $X$ is bought on the market, while knowledge is rented from a large set of suppliers, i.e., both from workers - who own the initial endowment $A_{0}$ - and from patent holders - who own the patents on knowledge supplied by $R \& D$-firms after the initial instant $t=0$.

As will become clearer in the following, the amount of raw labor needed to produce each firm's output amount, $Y_{i}$, is being supplied for free according to the firm's

\footnotetext{
${ }^{9}$ See, e.g., pp. 428-429 in Acemoglu (2009).

${ }^{10} \mathrm{~F}$-firms cannot re-rent knowledge or share it with other firms; that is, no arbitrage is allowed.
} 
knowledge demand.

Assumption 3.3. $F(\cdot, \cdot)$ is concave and linearly homogenous, with $F_{1}>0, F_{2}>0$, $F_{11}<0$ and $F_{22}<0$, where $F_{j}$ and $F_{j j}$ denote the first-order and second-order partial derivatives with respect to arguments $j=1,2$ respectively. Moreover, the standard Inada conditions hold for both arguments.

Consistently to the fact that each firm's maximization problem has only two decision variables, $X$ and $A$, Assumption 3.3 rules out increasing returns to scale at the level of the single firm. Assumption 3.3 also postulates decreasing returns to knowledge for the production function $F$. This can be rationalized by considering that the transfer of knowledge from the research sector to the final good's production involves a rescaling in order to take into account its decreasing effectiveness in terms of output augmentation, due, e.g., to partial substitution of previously used results with new knowledge.

Because households own the whole knowledge stock $A$ - the initial endowment $A_{0}$ plus the amount $\left(A-A_{0}\right)$ purchased from $R \& D$-firms - they are willing to supply the whole lot provided that they can earn a strictly positive price on it. Specifically, denoting by $A$ the aggregate demand for knowledge, by $A^{*}$ the whole endowment available in the economy and by $p^{A}$ the rental price of knowledge, the market clearing conditions for knowledge can be written in complementary slackness form as

$$
A \leq A^{*}, p^{A} \geq 0 \quad \text { and } \quad\left(A^{*}-A\right) p^{A}=0,
$$

which imply $A=A^{*}$ whenever $p^{A}>0$, as will be the case in the following. Indeed, in the instantaneous market equilibrium knowledge can be considered in fixed supply, as it is a stock variable: hence, at each instant, the equilibrium price $p^{A}$ depends only on demand. However, knowledge is a nonrival good; therefore, all $F$-firms can be supplied at the same time with the whole stock $A^{*}$ available, and the subscript $i$ can be dropped from the amount of knowledge employed in their production function.

Let us reformulate firm $i$ output as

$$
Y_{i}=A L_{i} f\left(\frac{x}{A}\right), \quad \text { with } f(\cdot)=F(\cdot, 1)
$$

where $^{11} x=X_{i} / L_{i}$ is the per capita intermediate good. As Assumption 3.3 envisages linear homogeneity, the scale and the number of firms are indeterminate, so that we can assume without loss of generality that at each instant $t$ there is a large number of firms, say $M(t)$, and several, say $N(t)<M(t)$, output levels $Y_{i}(t)$, for $i=1, \ldots, N(t)$, each corresponding to a different amount $L_{i}$ of raw labor employed, that are being produced by $m_{i}(t)$ identical firms operating at the same level, with $m_{i}(t) \geq \underline{m}>0$ where $\underline{m}$ is a number sufficiently large to sustain a competitive market. Therefore, at each instant $t$ the economy is populated by $M(t)=\sum_{i=1}^{N(t)} m_{i}(t)$ firms producing a total amount $Y(t)=\sum_{i=1}^{N(t)} m_{i}(t) Y_{i}(t)$ of final consumption good. ${ }^{12}$ If $N(t)=1$ then $M(t)=m_{1}(t)$ and $Y(t)=m_{1}(t) Y_{1}(t)$. Knowledge suppliers can observe the firms' size and thus tell apart each $m_{i}(t)$ group of firms.

${ }^{11}$ All firms employ the same intermediate good/labor ratio, $x \equiv x_{i}=X_{i} / L_{i}$, as will become clear in the sequel. ${ }^{12}$ Alternatively, one can assume that there is a continuum of output levels $Y(i, t) \geq 0, i \in[0, N(t)]$, each produced by a density $m(i, t) \geq 0$ of identical $F$-firms, so that the economy is populated by an absolutely continuous distribution of firms over the compact support $[0, N(t)]$, and total output is given by $Y(t)=$ $\int_{0}^{N(t)} m(i, t) Y(i, t)$ di. Note that when $m(i, t)>0$ there is a continuum of firms each producing $Y(i, t)$, thus assuring that such sub-market is competitive, while if $m(i, t)=0$ there are no firms producing the $Y(i, t)$ level. 
The FOC for each $F$-firm's profit maximization are:

$$
\begin{gathered}
\frac{\partial Y_{i}}{\partial X_{i}}=f^{\prime}\left(\frac{x}{A}\right)=1 \\
\frac{\partial Y_{i}}{\partial A}=L_{i}\left[f\left(\frac{x}{A}\right)-\frac{x}{A} f^{\prime}\left(\frac{x}{A}\right)\right]=L_{i} \gamma\left(\frac{x}{A}\right)=p_{i}^{A} .
\end{gathered}
$$

Condition (5) holds because the intermediate goods are priced at the numeraire, while in condition (6) the term $\gamma(x / A)$ denotes the equilibrium royalty per augmented worker, which depends on the ratio $x / A$. Condition (6) shows that the equilibrium price for knowledge, $p_{i}^{A}$, is the firm $i$ demand price evaluated at the given amount $A=A^{*}$.

\subsection{Lindahl Prices}

As there are many $F$-firms operating at each output level $Y_{i}$, one can identify $N(t)$ independent and competitive sub-markets for knowledge. In each sub-market, the demand price is scaled by the raw labor amount $L_{i}$ needed to produce its representative firm specific output size, $Y_{i}$, according to (4). Because $A$ is nonrival and inelastically supplied, all the sub-markets clear at the same amount $A=A^{*}$. However, because $F$-firms demand prices are scaled according to $L_{i}$, the knowledge equilibrium rental price in each sub-market will differ accordingly; in other words, $p_{i}^{A}=L_{i} \gamma(x / A)$ are Lindahl prices. ${ }^{13}$ Households who invested in new patented knowledge receive payments from all the firms in the economy and earn a share of total output, $Y$, given by $L \gamma(x / A)\left(A-A_{0}\right) / Y$, while, in their role of workers who rent $A_{0}$ to all $F$-firms, households receive $L \gamma(x / A) A_{0} / Y^{14}$

Figure 1 shows how Lindahl prices form for two hypothetical sub-markets $i=1,2$. Without loss of generality, suppose that there is a continuum of firms in each submarket, both with mass $m_{1}=m_{2}=1$. While all the firms in each sub-market have the same size, sub-market 1 is populated by firms which are smaller in size than those operating in sub-market 2 , that is, $Y_{1}<Y_{2}$, so that, according to (4), $L_{1}<L_{2}$. Lindahl prices $p_{i}^{A}=L_{i} \gamma\left(x / A^{*}\right)$, for $i=1,2$, are determined by the intersection of the marginal products of knowledge given by $\partial Y_{i} / \partial A=L_{i} \gamma(x / A)$ - which, for fixed $L_{i}$ and $x=X_{i} / L_{i}$, is the representative firm's demand for knowledge in the $i^{t h}$ submarket ${ }^{15}$ as a function of the only variable $A$-with the amount $A^{*}$ in fixed supply. According to Assumption 3.3, the demand functions $L_{i} \gamma(x / A)$ are decreasing in $A$ when $L_{i}$ and $x=X_{i} / L_{i}$ are kept constant. The upper black demand curve in Figure 1 refers to the whole knowledge market; it is obtained as the "vertical sum" of the sub-markets' demands, yielding the sum of Lindahl prices for knowledge in the whole economy for any given $A$. At the intersection point of the total market demand with the amount $A^{*}$ inelastically supplied, the royalty for knowledge - that is, the Lindahl price of the whole lot $A^{*}$-is determined as $p^{A}=L \gamma\left(x / A^{*} x\right)$, where $L=L_{1}+L_{2}$.

In Figure 1 also the inelastic supply of the basic knowledge endowment, $A_{0}$, is

\footnotetext{
${ }^{13}$ A similar approach has been pursued in Chantrel, Grimaud, and Tournemaine (2012) with reference to knowledge demanded by producers of differentiated goods, each one with its specific sub-market and Lindahl price. They also show that under perfect information and full excludability this is the only market equilibrium. ${ }^{14}$ See Example 1 in the working paper version of this paper (Marchese and Privileggi 2016) for an illustration on how Lindahl prices are determined when production is Cobb-Douglas.

${ }^{15}$ As $m_{1}=m_{2}=1$, in Figure 1 the representative firm's demand coincides with the total sub-market demand.
} 


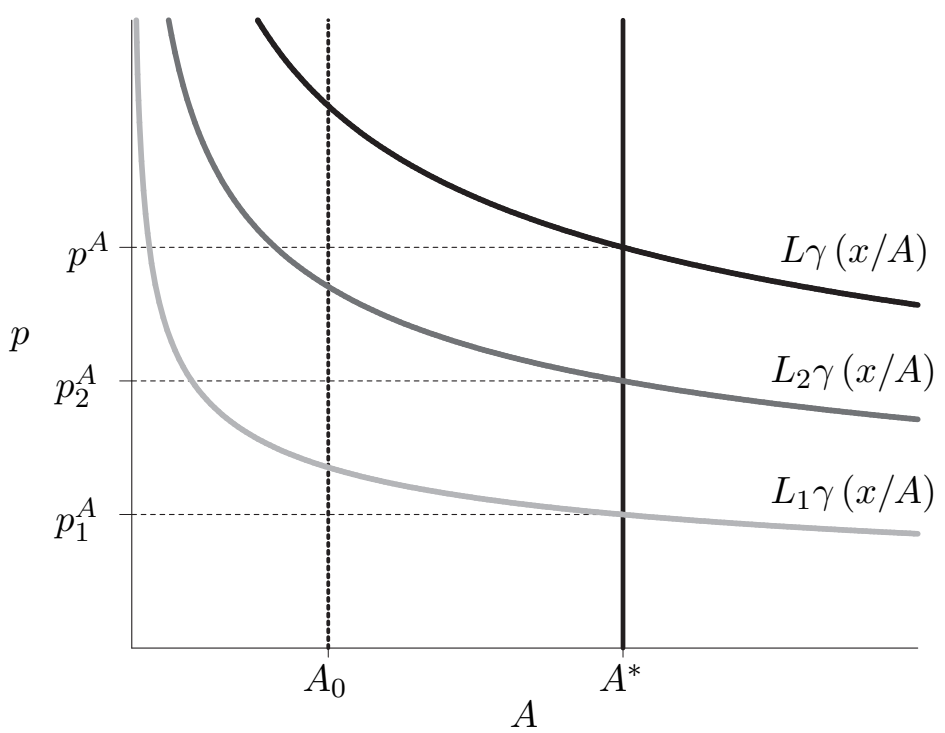

Figure 1. Equilibrium Lindahl prices in the two sub-markets, $p_{1}^{A}, p_{2}^{A}$, and in the whole knowledge market, $p^{A}$, as the intersection between sub-markets knowledge demand functions $L_{1} \gamma(x / A), L_{2} \gamma(x / A)$, and the whole demand function $L \gamma(x / A)$, with the inelastic supply function $A \equiv A^{*}$.

plotted as a dashed vertical line to the left of the total supply $A^{*}$. Denoting by

$$
h=A_{0} / L
$$

the (constant) amount of initial knowledge endowment that can figuratively be assigned to each agent in proportion to its share of property rights, ${ }^{16}$ and after conveniently rescaling $L$ through factor $h, A_{0}$ can alternatively be interpreted as the inelastic raw labor supply $A_{0}=h L$. Hence, when the knowledge market is in equilibrium-i.e., when the whole $A_{0}$ is rented at the royalty $p^{A}=\left(L_{1}+L_{2}\right) \gamma\left(x / A^{*}\right)$-also the total labor force, which is jointly supplied, must be employed.

Lindahl prices turn out to be proportional to labor employed by each firm $i$, that is:

$$
\frac{p_{i}^{A}}{p^{A}}=\frac{L_{i} \gamma\left(x / A^{*}\right)}{\left(L_{1}+L_{2}\right) \gamma\left(x / A^{*}\right)}=\frac{L_{i}}{L}
$$

Thus, an equilibrium of the market for knowledge also implies an allocation of workers to each firm corresponding to its relative Lindahl price. This allocation is viable because it implies that each worker receives the same compensation $\gamma\left(x / A^{*}\right) A_{0}$ and is thus properly compensated according to her share $1 / L$ of ownership of each idea belonging to the basic knowledge endowment $A_{0}$. Hence, in this decentralized equilibrium firms both pay a price for knowledge and employ a number of workers corresponding to their size in terms of final goods' production (or, in other words, according to the sub-market to which they belong), and both knowledge and raw labor are fully and efficiently allocated.

While in general the resort to Lindahl prices for public goods is deemed unattainable due to the lack of proper information, in this case the observable size of each firm

${ }^{16}$ Note that agents share the ownership of the cultural heritage, which is collective and not excludable for them. Property rights on subsets of $A_{0}$ cannot be assigned. 
renders it possible to ascribe it to a specific sub-market, and all the suppliers of knowledge can easily identify such sub-markets and the demand arising therein. The demand revelation problem is in general less severe when the public good is an input than when it is a consumption good, as demand in the former case derives from the profit function while in the latter it derives from the utility function which has a lower degree of measurability (Dasgupta 2001).

According to (5) and (6) the per capita product $y_{i}=Y_{i} / L_{i}=A f(x / A)$ is fully distributed to the per capita intermediate good, $x=X_{i} / L_{i}$, and knowledge, $A$, that is,

$$
y_{i}-x-\gamma(x / A) A=A f(x / A)-f^{\prime}(x / A) x-A f(x / A)+x f^{\prime}(x / A)=0,
$$

so that each firm (and thus the whole industry) makes no profit. Households, either in their role of workers as suppliers of $A_{0}$ or as holders of the patents covering $\left(A-A_{0}\right)$, are paid according to their entitlements. Because in the market for final goods all firms face the same rental price for intermediate goods and face Lindahl prices for knowledge, all use the same combination of intermediate goods and knowledge-augmented labor. We thus refer in the following to a representative firm and drop the subscript $i$.

\subsection{A Rationale for the Absence of Raw Labor Compensation}

Our approach relies on the assumption of rational expectations, where all agents make all their plans on the basis of expectations about future prices. In our model positive prices for both labor and knowledge are not sustainable under competition, since the production function of final goods $Y_{i}=F\left(X_{i}, A_{i} L_{i}\right)$ implies that the output is exhausted whenever, in addition to the intermediate good $X$, either knowledge or raw labor are paid according to their marginal product. Hence, provided that only one of these two prices can be positive, we now argue that workers turn out to be indifferent between which one it is, while the household, as the owner of the whole $A$ and beneficiary of technical progress, prefers a positive price for knowledge.

Indeed, at instant $t=0$ both workers and firms are indifferent between a compensation based on the marginal value of either raw labor or knowledge. To see this, recall from (6) that $\partial Y_{i} / \partial A=L_{i} \gamma(x / A)$, while differentiating the production function (4) with respect to $L_{i}$ yields the marginal product of raw labor as $\partial Y_{i} / \partial L_{i}=A\left[f(x / A)-(x / A) f^{\prime}(x / A)\right]=A \gamma(x / A)$, from which we infer that only the scale in which the marginal product is measured changes from $L_{i}$ to $A$. The effect of this change of scale is cancelled out, however, whenever the total compensation received by all workers is calculated as if they were paid for raw labor, as $\sum_{i=1}^{N(t)} m_{i}(t) L_{i} A \gamma(x / A)=A L \gamma(x / A)=A p^{A}$, where $p^{A}=\sum_{i=1}^{N(t)} m_{i}(t) L_{i} \gamma(x / A)=$ $\operatorname{L\gamma }(x / A)$ is the total payment based on Lindahl prices.

As long as workers accept of being paid according to their knowledge endowment from the outset, their total compensation stays put forever at $A_{0} L \gamma\left(x / A_{0}\right)$. On the other hand, the total compensation accruing to knowledge changes through time and is given by $A_{t} L \gamma\left(x / A_{t}\right)$. In the following Section 4 it will be shown that $\gamma\left(x / A_{t}\right)$ turns out to be constant through time, so that, whenever the knowledge stock keeps piling up as time elapses and the economy exhibits sustained growth, $A_{t} L \gamma\left(x / A_{t}\right) \geq A_{0} L \gamma\left(x / A_{0}\right)$ holds for all $t>0$. However, while only patents' owners benefit from such increase, from the raw labor supply perspective being paid as owners of knowledge $A_{0}$ let workers not worse-off than being paid for the supply of $L$; this 
is because the implication in the latter case is that no knowledge advance can occur after the initial instant $t=0$, as a zero compensation left to knowledge annihilates any incentive to innovate by $R \& D$-firms. Therefore, the stagnation of total labor income at $A_{0} L \gamma\left(x / A_{0}\right)$ would occur all the same.

As we are interested in describing a growing competitive economy in which there is a market supply of inventions, we assume that firms demand knowledge $A$ from the outset and workers are willing to supply $A_{0}$ joint with raw labor.

The absence of a specific price for raw labor does not imply efficiency losses, because raw labor is inelastically supplied and efficiently allocated. One might also liken knowledge-augmented labor $A L$ to human capital, but in our model the scale through which human capital is evaluated is provided by the embodied knowledge contents, which, notwithstanding such embodiment, are still homogeneous with respect to the dematerialized contents protected by patents. Because in our economy the dematerialization process is prevailing, only the basic human capital - embedding just the initial knowledge endowment $A_{0}$-is demanded.

One implication of the model is that the benefits of technological progress are funneled to the owners of the intangible capital protected by patents, while workers' compensation is stagnating as it is linked to the original knowledge endowment. The negative consequence of the model with respect to workers' income takes to the extreme a tendency that might be inferred from the stylized facts pertaining to the long term evolution of income shares. Corrado, Hulten, and Sichel (2009), e.g., show that labor's income share decreased significantly over the last 50 years in the US, provided that investments in intangibles and their income share are properly accounted for. The negative effects implied by our model on workers' income might be mitigated if one assumed that the role of worker and that of new knowledge provider can overlap. With an increasing common cultural endowment ${ }^{17}$ the income share accruing to workers would increase.

\section{Intertemporal Competitive Equilibrium}

Definition 4.1. A feasible allocation in this economy is a set of time paths of consumption levels $[C(t)]_{t=0}^{\infty}$, intermediate goods' flows $[X(t)]_{t=0}^{\infty}$, aggregate $\mathrm{R} \& \mathrm{D}$ expenditures $[J(t)]_{t=0}^{\infty}$, and knowledge stock levels $[A(t)]_{t=0}^{\infty}$, such that, at each instant $t \geq 0$, the aggregate resource constraint, $C(t)+X(t)+J(t) \leq F[X(t), A(t) L]$, is satisfied, while new knowledge production occurs according to $\dot{A}(t) \eta(t) \leq J(t)$, where $\eta(t)$ is the amount of final goods technically needed to produce 1 unit of $A(t)$.

In order to incentivize all households to invest in new knowledge production, while keeping the knowledge market in intertemporal equilibrium, under Assumption 3.2 the following free-entry condition must hold:

$$
V(t)=\int_{t}^{+\infty} \gamma(v) L e^{-\int_{t}^{v} r(s) \mathrm{ds}} \mathrm{dv}=\eta .
$$

It postulates that the present value $[r(t)$ is the instantaneous interest rate] of future royalties, $\Gamma(t)=\gamma(t) L$, where $\gamma(t)$ denotes the equilibrium royalty per worker at instant $t$, must be equal to the (constant) production cost $\eta$ of a unit of new knowledge.

${ }^{17}$ In Cozzi (1998) the dynamic of such common cultural endowment is endogenized by a market for nontechnical education. 
Because the only asset in the economy is expressed in terms of the knowledge stock $A$ owned by households, a first consequence of (7) is that $B(t)=V(t) A(t)=\eta A(t)$ must hold.

Definition 4.2. An equilibrium is a feasible allocation in which the evolution of $[A(t)]_{t=0}^{\infty}$ is determined by free entry in a competitive market for $\mathrm{R} \& \mathrm{D}$, the evolution of $[r(t)]_{t=0}^{\infty}$ is consistent with the free entry property, the evolution of $[C(t)]_{t=0}^{\infty}$ is consistent with household maximization, both $R \& D$-firms and $F$-firms maximize their profit, Lindahl prices $\left[p_{i}^{A}(t)\right]_{t=0}^{\infty}$ clear each sub-market for knowledge when all $F$-firms use the whole available stock $A$ and the optimal number of workers $L_{i}$, the royalties $\left[p^{A}(t)\right]_{t=0}^{\infty}$ clear the whole market for knowledge with full employment of raw labor. In other words, in equilibrium at each instant $t \geq 0, J(t)$ is consistent with (7) while $[C(t), r(t)]$ satisfy the Euler equation (2) and the transversality condition $\lim _{t \rightarrow+\infty} B(t) e^{-r t}=\lim _{t \rightarrow+\infty} \eta A(t) e^{-r t}=0$ holds.

As, under Assumption 3.3, the derivative of function $f$ defined in (4) is decreasing, $f^{\prime}$ is invertible and from (5) we get the demand for the intermediate good $x$ by the $F$-firms, which turns out to be linear in $A$ :

$$
x=\delta A, \quad \text { with } \delta=\left(f^{\prime}\right)^{-1}(1) .
$$

Thus, $\delta$ is a constant uniquely defined by the choice of the production function $F(\cdot, \cdot)$. As the ratio $\delta \equiv x / A$ is constant, from (6) we obtain the per capita willingness to pay for knowledge, which turns out to be constant as well:

$$
\gamma\left(\frac{x}{A}\right) \equiv \gamma=f(\delta)-\delta f^{\prime}(\delta)=f(\delta)-\delta
$$

where in the third equality we used the definition of $\delta$ in (8).

Hence, we can use the fact that, according to (9), $\gamma$ is constant and differentiate both sides in (7) with respect to $t$ to get

$$
\dot{V}(t)=r(t) \int_{t}^{+\infty} \gamma L e^{-\int_{t}^{v} r(s) \mathrm{ds}} \mathrm{dv}-\gamma L=0,
$$

which implies that the present value of future royalties does not change in time and, after substituting the integral with (7), the equilibrium interest rate regulating the transfer of wealth through time (via the only state variable, which is the knowledge $A)$ is constant as well, $r(t) \equiv r$, and given by

$$
r=\frac{\gamma L}{\eta}
$$

In order to use (2) to look for a balanced growth path (BGP) type of equilibrium, we must assume that $1 / \varepsilon_{u}(C)=1 / \sigma$ is a constant, ${ }^{18}$ with $\sigma>0$. Hence, using (10) in (2) we obtain the following constant rate of growth of consumption, $C$, knowledge,

${ }^{18}$ If we are interested in an asymptotic balanced growth path (ABGP) it is sufficient to assume that $\lim _{t \rightarrow+\infty}\left[1 / \varepsilon_{u}(C)\right]=\lim _{t \rightarrow+\infty}\left\{-u^{\prime}(C) /\left[u^{\prime \prime}(C) C\right]\right\}=1 / \sigma$. This approach will be pursued in Section 5 . 
$A$, and output, $Y$, along the BGP:

$$
g=\frac{\dot{C}}{C}=\frac{\dot{A}}{A}=\frac{\dot{Y}}{Y}=\frac{1}{\sigma}\left(\frac{\gamma L}{\eta}-\rho\right) .
$$

Proposition 4.3. Suppose that Assumptions 3.1-3.3 hold and the intertemporal elasticity of substitution is constant-i.e., $\varepsilon_{u}(C)=-\left[u^{\prime \prime}(C) C\right] / u^{\prime}(C) \equiv \sigma>0$. Then, if

$$
\gamma L>\rho \eta \quad \text { and } \quad(1-\sigma) \gamma L<\rho \eta,
$$

the economy admits a unique BGP along which knowledge, output, and consumption all grow at the same rate $g>0$ given by (11). Moreover, there are no transition dynamics: the economy immediately jumps on the BGP starting from $t=0$.

The novelty introduced by assuming that $F$-firms, operating under constant returns to scale, pay for the use of knowledge through Lindahl prices both to compensate patent holders and suppliers of basic knowledge joint with raw labor, together with Assumption 3.2 of a constant cost for new knowledge production, allows for the solution characterized in Proposition 4.3 to be Pareto optimal even in a totally decentralized setting.

Proposition 4.4. The BGP equilibrium characterized in Proposition 4.3 is Pareto optimal.

Three features of our model are crucial to explain Proposition 4.4.

(1) As knowledge is paid its Lindahl price there is no room for monopolistic power exploitation in the economy as in the standard literature, that is, nowhere markups are being applied. Actually, one can read (7) as a dynamic version of the famous Samuelson rule for public goods, since it establishes the equality between the present value of the sum (the integral) of the marginal benefits of knowledge and the marginal cost of knowledge production. This implies that knowledge is efficiently provided, as the same choices that a social planner would have made with respect to knowledge accumulation emerge in a decentralized setting.

(2) The stagnation of workers' income, due to the fact that knowledge increases are provided only by patent holders, would be particularly problematic in an economy in which, unlike that described in this paper, the population were composed by two groups: workers (endowed only with labor $L$ and $A_{0}$ ), who consume their all income, and holders of the remaining assets, who consume and save. While we leave for future research the study of this case, one can guess that in this economy an evolution of income shares according to the pattern described so far is likely to prompt protests against the raising income inequality and give rise to social pressure for redistributive policies.

(3) Finally, Assumption 3.2, by postulating a constant unit cost $\eta$ for the production of new ideas, rules out intertemporal knowledge spillovers or other types of externalities. Our next step is to relax this assumption. 


\section{Non Constant Cost of Knowledge Production}

An example of a well-known model in which the cost of knowledge production turns out to be constant like in our simple setting discussed in the previous section is the celebrated original contribution by Romer (1990). However, as most of the major contributions that followed Romer's seminal paper confirm, it is widely accepted that Assumption 3.2 introduces a definitely unrealistic restriction. For instance, Tsur and Zemel (2007) consider a continuous-time version of the original model by Weitzman (1998) in which knowledge evolves according to a recombinant technology characterized by a variable unit cost of knowledge production,

$$
\eta(t)=\varphi[A(t)]
$$

in which the unit cost function $\varphi(\cdot)$ indirectly depends on time through the knowledge stock $A(t)$ evolution. The recombinant knowledge production function by Weitzman is well suited for our approach because it assumes that its main input is expressed in terms of financial resources, ${ }^{19} J(t)$. Hence, while we keep the assumption of Section 3 that there is no aggregate uncertainty in the innovation process, in this section we allow the unit cost of knowledge production, $\eta$, to vary over time.

Assumption 5.1. Each new idea can be produced at a time-dependent unit cost, $\eta(t)>0$.

According to Assumption 5.1, define the innovation possibilities frontier as

$$
\dot{A}(t)=\frac{J(t)}{\eta(t)},
$$

in which time-dependence has been emphasized. ${ }^{20}$

From the representative household optimization problem (1) we get the same necessary Euler condition as in (2). Also, nothing changes from the point of view of knowledge demand: the per augmented worker royalty is still constant and, according to (9), given by $\gamma(x / A) \equiv \gamma=f(\delta)-\delta$. Only the free-entry condition (7) changes, as now the RHS depends on time:

$$
V(t)=\int_{t}^{+\infty} \gamma(v) L e^{-\int_{t}^{v} r(s) \mathrm{ds}} \mathrm{dv}=\eta(t)
$$

Differentiating it with respect to time leads to

$$
\dot{V}(t)=r(t) \int_{t}^{+\infty} \gamma L e^{-\int_{t}^{v} r(s) \mathrm{ds}} \mathrm{dv}-\gamma L=\dot{\eta}(t)
$$

\footnotetext{
${ }^{19}$ As a matter of fact, all mainstream extensions of Romer's model implicitly assume time-dependent costs of producing new ideas. In such a framework, increasing research costs can even offset scale effects (Cozzi and Spinesi 2004). However, like Romer's one, all these models are based on knowledge production functions that use labor as a main input factor, so that the cost of new knowledge depends on the equilibrium wage. Because, according to (3) and (14), in our setting we assume that knowledge is produced through financial investment rather than labor, these contributions are not directly comparable with our model.

${ }^{20}$ Note that (14) encompasses also the case in which new knowledge is being produced by decentralized $R \& D$ firms for a price $\eta(t)=\psi[A(t)]$ that includes a mark-up over the Tsur and Zemel (2007) first-best cost $\varphi[A(t)]$, as in Marchese et al. (2017).
} 
which, after substituting the integral with (15), yields the interest rate

$$
r(t)=\frac{\gamma L}{\eta(t)}+\frac{\dot{\eta}(t)}{\eta(t)} .
$$

Note that, although the total royalty $\Gamma=\gamma L$ remains constant, under Assumption 5.1 the interest rate varies in time according to the law of motion of $\eta(t)$.

Because from (15) $V(t)=\eta(t)$, equation (16) can be rewritten in the familiar form of a Hamilton-Jacobi-Bellman equation,

$$
r(t) V(t)=\gamma L+\dot{V}(t),
$$

in which the evolution through time of knowledge's value takes into account the assets' gains/ losses $\dot{V}(t)=\dot{\eta}(t)$ due to variations in the new knowledge's cost $\eta(t)$.

Using (16) in (2) we obtain the following time-dependent growth rate of consumption:

$$
g(t)=\frac{\dot{C}(t)}{C(t)}=\frac{1}{\varepsilon_{u}[C(t)]}\left[\frac{\gamma L}{\eta(t)}+\frac{\dot{\eta}(t)}{\eta(t)}-\rho\right] .
$$

As, by construction, this version of the model exhibits transition dynamics, we look for an Asymptotic Balanced Growth Path (ABGP) type of equilibrium. To this aim, we assume that asymptotically the intertemporal elasticity of substitution becomes constant, while the growth rate of the unit cost of new knowledge is required to vanish in the long-run; that is, we set $\lim _{t \rightarrow+\infty}\left[1 / \varepsilon_{u}(C)\right]=1 / \sigma, \sigma>0$, and $\lim _{t \rightarrow+\infty}[\dot{\eta}(t) / \eta(t)]=0$. Note that this setting is sufficiently general to encompass any type of new knowledge production function, envisaging either increasing or decreasing $\eta(t)$ along transition dynamics, while asymptotically the unit cost of new knowledge must converge to some positive constant, $\lim _{t \rightarrow+\infty} \eta(t)=\eta^{*}>0$, in order to satisfy the transversality condition for a solution of problem (1).

Proposition 5.2. Under Assumptions 3.1, 3.3 and 5.1, assume that $\lim _{t \rightarrow+\infty}\left[1 / \varepsilon_{u}(C)\right]=1 / \sigma, \sigma>0$, and $\lim _{t \rightarrow+\infty} \eta(t)=\eta^{*}>0$. Then, if

$$
\gamma L>\rho \eta^{*} \quad \text { and } \quad(1-\sigma) \gamma L<\rho \eta^{*},
$$

then the economy admits a unique ABGP along which knowledge, output, and consumption all grow at the same asymptotic growth rate given by

$$
g^{*}=\frac{\dot{C}}{C}=\frac{\dot{A}}{A}=\frac{\dot{Y}}{Y}=\frac{1}{\sigma}\left(\frac{\gamma L}{\eta^{*}}-\rho\right) .
$$

Conditions (19) and the growth rate (20) are the same as those in (12) and (11) respectively, only with the asymptotic value $\eta^{*}$ in place of the constant unit cost $\eta$. Along the transition dynamics the consumption growth rate $g$ in (18) may be either larger or smaller than its asymptotic value $g^{*}$ in (20), depending on the (transition) intertemporal elasticity of substitution $1 / \varepsilon_{u}(C)$, the (transition) knowledge cost $\eta$ and the sign of $\dot{\eta} / \eta$, that is, on whether building new knowledge involves increasing or decreasing costs as time elapses. 
When the cost of knowledge varies through time depending on the evolution of the knowledge stock, externalities arise, but the households are not able to keep them into account in their decision process, as they can only observe the ensuing price changes, $\dot{\eta}(t)$, embedded in the interest rate according to (16). Clearly, under these circumstances the equilibrium described in Proposition 5.2 turns out to be not Pareto optimal, as we now show by solving the social planner problem associated to (1).

Now we assume that the unit cost of new knowledge production $\eta(t)$ has the form in (13) and, as in Proposition 5.2, $\lim _{t \rightarrow+\infty} \eta(t)=\lim _{t \rightarrow+\infty} \varphi[A(t)]=\eta^{*}>0$ when there is knowledge growth, $\dot{A}(t) / A(t)>0$. Following the same argument as in the proof of Proposition 4.4 in the Appendix, the social planner first maximizes net output, $Y(t)-X(t)=A(t) L f[x(t) / A(t)]-x(t) L$, with respect to $x(t)$, obtaining the optimal net output $Y^{S}(t)-X^{S}(t)=\gamma L A(t)$. Then, she maximizes the representative household's lifetime discounted utility as in (1) subject to the resource constraint $\gamma L A(t)=C(t)+J(t)$, which, according to (13) and (14), can be written as

$$
\dot{A}(t)=\frac{\gamma L A(t)-C(t)}{\varphi[A(t)]} .
$$

Denoting by $\lambda(t)$ the costate variable associated to the unique dynamic constraint (21) and dropping the time argument for simplicity, the current-value Hamiltonian of the social planner problem is

$$
H(A, C, \lambda)=u(C)+\lambda \frac{\gamma L A-C}{\varphi(A)} .
$$

Necessary conditions are

$$
\begin{gathered}
u^{\prime}(C)=\frac{\lambda}{\varphi(A)} \\
\dot{\lambda}=\rho \lambda-\lambda \frac{\gamma L \varphi(A)-(\gamma L A-C) \varphi^{\prime}(A)}{[\varphi(A)]^{2}} \\
\lim _{t \rightarrow+\infty} \lambda(t) A(t) e^{-\rho t}=0,
\end{gathered}
$$

where $(24)$ is the transversality condition. Differentiating with respect to time (22) one gets

$$
\frac{\dot{\lambda}}{\lambda}=\frac{\varphi^{\prime}(A) \dot{A}}{\varphi(A)}-\varepsilon_{u}(C) \frac{\dot{C}}{C}
$$

where $\varepsilon_{u}(C)$, as usual, denotes the inverse of the intertemporal elasticity of substitution. Coupling (25) with (23), using (21) and rearranging terms we obtain the following transitory consumption growth rate:

$$
g^{S}(t)=\frac{\dot{C}(t)}{C(t)}=\frac{1}{\varepsilon_{u}[C(t)]}\left\{\frac{\gamma L}{\varphi[A(t)]}-\rho\right\} .
$$

Also this version of the model exhibits transition dynamics; thus, we again look for an ABGP type of equilibrium which turns out to be the same as that characterized in Proposition 5.2. 
Proposition 5.3. Suppose that Assumptions 3.1, 3.3 and 5.1 with the specification in (13) hold. Moreover assume that $\lim _{t \rightarrow+\infty}\left\{1 / \varepsilon_{u}[C(t)]\right\}=1 / \sigma, \sigma>0$, and $\lim _{t \rightarrow+\infty} \varphi[A(t)]=\eta^{*}>0$ whenever there is positive knowledge growth, $\dot{A} / A>0$. Then, if conditions (19) hold, the social planner economy admits a unique ABGP along which knowledge, output, and consumption all grow at the same asymptotic growth rate characterized by the common constant growth rate as in (20) of Proposition 5.2 for the decentralized economy.

Furthermore, along the transition dynamics the consumption growth rate in (26) can be either larger or smaller than that in (18), specifically, $g^{S}>g$ when $\varphi^{\prime}[A(t)]<0$, while $g^{S}<g$ when $\varphi^{\prime}[A(t)]>0$.

From Proposition 5.3 we conclude that, asymptotically, the equilibrium in the decentralized model converges to the Pareto optimal solution. However, along the transition dynamics the consumption growth rate under social planner supervision in (26) can be either larger or smaller than that in the decentralized market economy in (18), depending on the sign of $\varphi^{\prime}[A(t)]$ which, in turn, determines the sign of the term $\dot{\eta}(t) / \eta(t)=\varphi^{\prime}[A(t)] \dot{A}(t) / \varphi[A(t)]$ in the square bracket of the RHS in (18). Indeed, a social planner controls the whole evolution of the knowledge stock $A(t)$ so that, with a unit cost of new knowledge production as in (13), the externalities of investments in knowledge leading to changes in $\varphi[A(t)]$ through time are now taken into account.

Specifically, with costs that decrease in time, $\varphi^{\prime}[A(t)]<0$, the growth rate in the transitional period is larger under central control than in the decentralized model: $g^{S}>g$. This is due to the presence of a positive externality, that is, when knowledge costs are decreasing in time, it becomes possible to produce subsequent inventions by subtracting less and less resources from other uses. This external effect, however, is not accounted for by private investors, while it is being considered by the central planner, who, accordingly, chooses a larger growth rate $g^{S}$ in the transitional period.

Conversely, if $\varphi^{\prime}[A(t)]>0$, the growth rate in the transitional period is smaller under the social planner supervision than in the decentralized model: $g^{S}<g$.

As inefficiencies arise in the transitional period under decentralization, corrective policy interventions might be designed. That is, a subsidy to $\mathrm{R} \& \mathrm{D}$ investments along the transition when $\varphi^{\prime}[A(t)]<0$ (a tax when $\varphi^{\prime}[A(t)]>0$ ) could be introduced to align the behavior of decentralized agents to the path envisaged by the social planner. We do not pursue here the detailed specification of such tools. It is interesting to note, however, that, as long as a "stepping on toes effect" prevails (i.e., $\left.\varphi^{\prime}[A(t)]>0\right)$, then an alternative to taxation might be represented by a suitable time path of reductions of the extent of rights granted to patent holders. ${ }^{21}$ This would entail a corresponding expansion of the share of property rights on knowledge attributed to the cultural endowment of workers and thus a shift of compensations in favor of the latter. This approach could take care of efficiency (by suitably modulating the remuneration of investments in R\&D) and of equity, as the workers income share would increase. ${ }^{22}$

It is also evident from (11) and (20) that, as one expects, both the constant and the variable cost of knowledge production versions of our simplified economy exhibit the strong scale effect - the growth rate of the economy increases in population sizeas postulated by Jones $(1995 ; 1999 ; 2005)$ for knowledge-based endogenous growth models. This effect, which is not supported by empirical evidence, has prompted the

${ }^{21}$ This might correspond to the introduction of a limited duration of patents, an extension of the model that we leave for future research. We thank an anonymous referee for suggesting this direction of further investigation. ${ }^{22}$ See Section 6 in the working paper version of this paper (Marchese and Privileggi 2016) for a worked out example under different assumptions on the cost of knowledge production. 
elaboration of new models either semi-endogenous (as, e.g., in Jones 1995) or "second generation" endogenous (as, e.g., in Peretto 1998), or hybrid (Cozzi 2017). An extension of the model controlling for the scale effect in a framework of variable population is reported in the working paper version of this paper (Marchese and Privileggi 2016).

\section{Conclusions}

The model presented in this paper, in addition to being simple, is based on assumptions for the fundamentals of the economy that cover the broadest class of neoclassical environments. Specifically, unlike most of the existing literature (including some papers mentioned above) that requires peculiar functional forms like CIES (if not logarithmic) preferences for the representative consumer and Leontief or Cobb-Douglas technologies for the productive sector, our setting works for any increasing and concave utility function with constant asymptotic intertemporal elasticity of substitution and CRS production function exhibiting decreasing returns in each factor taken alone. Moreover, also the creation of new knowledge in the R\&D sector occurs according to a sufficiently general family of technologies that include those already considered in the literature; the only restriction is that these technologies asymptotically must envisage a constant unit cost of production.

In this setting we take into account the increasingly immaterial characteristics assumed by technological progress and the implications this has on the ways in which it is transferred to final production. On this basis we thus assume that both a direct use of ideas in final goods' production and the revelation of firm's willingness to pay for accessing knowledge arise. A further feature that characterizes our model is the reconsideration of the role of labor. In our scenario cognitive tasks - traditionally performed by medium or even high skilled workers - are embedded into routines and software, and are thus automated. The truly creative activities are done by research firms under the protection of IPR. As for the tasks traditionally performed by low-skilled workers, they too are considered as based on models or routines. Firms demand these intellectual contents " embedded" in raw labor. Thus, both workers and patent holders supply the same type of input - i.e., ideas, models of behavior, knowledge - while workers provide them jointly with raw labor. As knowledge growth occurs through investments in patented innovations, an important implication of the model is a tendency toward the compression of incomes paid to workers - which can reduce to the sole compensation of the basic competencies - while the remaining income goes to patent holders.

We show that when one takes into account the aforementioned trends of recent technological progress, competition becomes viable. Hence the economy can reach the first best if knowledge, while being nonrival, is homogeneous and fully excludable when used in final goods production. Even if partial excludability occurs, second best results would be confined to the transitional period, while first best is reached all the same in the long run.

Idea-based competitive growth models routinely assume that the $\mathrm{R} \& \mathrm{D}$ activity is not protected by patents, so that the inventor cannot unduly restrict the exploitation of her invention by others. In fact, in Hellwig and Irmen (2001) a patent would allow the inventor/producer of final goods to permanently prevent the spillover of the productivity increases deriving from her invention and thus harm economic growth; in Boldrin and Levine (2008) knowledge does not exist outside the private and excludable goods in which it is embodied, so that granting a patent would simply allow the holder a distortionary monopolistic right, not needed to finance the initial cost of invention. 
In our basic model, instead, the patent is at the same time useful and harmless. It is useful because it gives the patent holder standard full property rights, so that the free exploitation of research results - as happens in the Hellwig and Irmen model after the information has leaked - cannot occur. Patents are harmless because the possibility of restricting the embedding rival good (e.g., the copies, as in Boldrin and Levine) does not arise, as knowledge is disembodied. Hence the property right granted by the patent just plays the role of allowing the holders of the stock of knowledge to be paid according to the latter's social marginal benefit. Limitations to the rights granted by patents, with a corresponding expansion of property rights on knowledge attributed to workers, however, can be in order in the transitional period when new knowledge production occurs under increasing costs. Stressing that competition is logically viable in a growing economy with endogenous knowledge creation has important policy implications, since it means that, e.g., regulatory and judicial interventions in the field of patents and IPR can foster competition without fearing that a collapse of research activities arises.

While the potential unwanted effects of technological progress have been often identified with the possible growth in unemployment due to the substitution of capital for labor, these dire effects did not materialize in the last decades in advanced countries, where unemployment - except for the financial crisis years - has not been the more worrying problem. One of the prominent social concerns has actually been instead the shrinking of labor income share. This paper provides a possible rationale for this stylized fact, which does not fit well into standard growth models where the stability of factors' income shares is a tenet.

\section{Appendix}

Proof of Proposition 4.3. Clearly, the first condition in (12) implies that, according to (11), $g=\dot{C} / C>0$. Differentiating with respect to time $\ln (Y)$, with $Y$ as in (4), and recalling that, from (8), $x / A \equiv \delta$ is constant, it is immediately seen that $\dot{Y} / Y=\dot{A} / A$. Recall that, from (7), $B=V A=\eta A$; hence, as under Assumption 3.2 both $\eta$ and, by (10), the interest rate, $r=\gamma L / \eta$, are constant the instantaneous budget constraint in (1) can be rewritten as

$$
\frac{\dot{A}}{A}=\frac{1}{\eta}\left(\gamma L-\frac{C}{A}\right)
$$

which implies that, in order to $\dot{A} / A$ be constant along the BGP, the ratio $C / A$ on the RHS must be constant as well, which is possible if and only if $\dot{A} / A=\dot{C} / C=g$. Next, note that the second condition in (12) implies that $r>g=\dot{A} / A$, so that the transversality condition for problem (1), $\lim _{t \rightarrow+\infty} B(t) e^{-r t}=\lim _{t \rightarrow+\infty} \eta A(t) e^{-r t}=$ 0 , holds. Finally, for each $A(t)$ the amount of the intermediate good is given by (8) as $x(t)=\delta A(t)$; therefore, in $t=0, x(0)=\delta A_{0}$ and the economy is immediately put on the BGP.

Proof of Proposition 4.4. The resource constraint of the economy at instant $t$ is

$$
C(t)+J(t)=Y(t)-X(t)
$$


where on the RHS we consider total output net of the intermediate goods, $X(t)=$ $x(t) L$. Dropping time dependency for simplicity, in order to obtain a dynamic constraint in the only variables $A$ (state) and $C$ (control) a social planner first considers maximization of the net output $Y-X=[A f(x / A)-x] L$ with respect to $x$ for a given stock $A$ at instant $t$ : the solution is $x^{S}=\left(f^{\prime}\right)^{-1}$ (1) $A=\delta A$, where the superscript " $S$ " denotes the level of per capita intermediate good chosen by the social planner, which happens to be the same as in (8). Hence, net output turns out to be $Y^{S}(t)-X^{S}(t)=L[f(\delta)-\delta] A(t)=\gamma L A(t)$, where in the last equality we used (9). Under Assumption $3.2 J=\eta \dot{A}$, and (28) can be rewritten as

$$
\dot{A}(t)=\frac{\gamma(t) L A(t)-C(t)}{\eta},
$$

which, as $B(t)=\eta A(t)$ with $\eta$ constant, turns out to be the same as the household's budget constraint in (1). Hence, the social planner problem is the same as (1) and has the same equilibrium of Proposition 4.3 as solution.

Proof of Proposition 5.2. The arguments are the same as in the previous proof of Proposition 4.3 and thus we omit them. The only difference is that now from (15) $B(t)=V(t) A(t)=\eta(t) A(t)$ holds, so that $\dot{B}(t)=\dot{\eta}(t) A(t)+\eta(t) \dot{A}(t)$; however, it is immediately seen that the instantaneous budget constraint in (1) at each instant $t$ remains the same as in (27), as, using (16) and rearranging terms,

$$
\frac{\dot{A}(t)}{A(t)}=\frac{\gamma L}{\eta(t)}+\frac{\dot{\eta}(t)}{\eta(t)}-\frac{\dot{\eta}(t)}{\eta(t)}-\frac{C(t)}{\eta(t) A(t)}=\frac{1}{\eta(t)}\left[\gamma L-\frac{C(t)}{A(t)}\right] .
$$

When $\lim _{t \rightarrow+\infty} \eta(t)=\eta^{*}>0, \dot{\eta}(t) \rightarrow 0$ as $t \rightarrow+\infty$ and, according to (16), $\lim _{t \rightarrow+\infty} r(t)=r^{*} \equiv \gamma L / \eta^{*}$; thus, the second condition in (19) implies that $r^{*}>$ $g^{*}=\dot{A} / A$, with $g^{*}$ defined in (20), so that the transversality condition for problem (1), $\lim _{t \rightarrow+\infty} \eta(t) A(t) e^{-r(t) t}=\lim _{t \rightarrow+\infty} \eta^{*} A(t) e^{-r^{*} t}=0$, holds.

Proof of Proposition 5.3. When $\dot{A} / A>0, \lim _{t \rightarrow+\infty} \varphi[A(t)]=\eta^{*}>0$ implies that $\lim _{t \rightarrow+\infty} \varphi^{\prime}[A(t)]=0$, so that it is immediately seen that, as $\lim _{t \rightarrow+\infty}\left[1 / \varepsilon_{u}(C)\right]=$ $1 / \sigma$, the consumption growth rate in (26) asymptotically converges to that defined in (20). As asymptotically the dynamic constraint (21) becomes equal to (27), the same argument as in the proofs of Proposition 4.3 applies to establish that $\dot{Y} / Y=\dot{A} / A=$ $\dot{C} / C=g^{*}$ while the first condition in (19) implies that, according to $(20), g^{*}>0$. The second condition in (19) is equivalent to $(1-\sigma) g^{*}<\rho$, which, according to $(25)$ and, under $(13), \lim _{t \rightarrow+\infty}[\dot{\eta}(t) / \eta(t)]=\lim _{t \rightarrow+\infty}\left\{\varphi^{\prime}[A(t)] \dot{A}(t) / \varphi[A(t)]\right\}=0$, implies

$$
\lim _{t \rightarrow+\infty}\left(\frac{\dot{\lambda}}{\lambda}+\frac{\dot{A}}{A}\right)=\lim _{t \rightarrow+\infty}\left(\frac{\dot{\eta}}{\eta}-\sigma \frac{\dot{C}}{C}+\frac{\dot{A}}{A}\right)=(1-\sigma) g^{*}<\rho,
$$

thus ensuring that the transversality condition (24) holds. 


\section{References}

Acemoglu, D. 2009. Introduction to modern economic growth. Princeton, NJ: Princeton University Press.

Acemoglu, D., and D. Autor, 2011. "Skills, tasks and technologies: implications for employment and earnings". In Handbook of labor economics Vol. 4B, edited by D. Card and O. Ashenfelter, 1043-1171. Amsterdam: Elsevier.

Acemoglu, D., and P. Restrepo. 2016. "The race between machine and man: implications of technology for growth, factor shares and employment". NBER Working Paper No. 22252.

Aghion, P., and P. Howitt, 1998. Endogenous growth theory. Cambridge, MA: MIT Press.

Arrieta Ibarra, I., L. Goff, D. Jiménez Hernández, J. Lanier, E. Glen Weyl, 2018. "Should we treat data as labor? Moving beyond 'free"'. American Economic Association Papers \&6 Proceedings 108: 38-42.

Boldrin, M., and D. K. Levine, 2008. "Perfectly competitive innovation". Journal of Monetary Economics 55 (3): 435-453.

Chantrel, É., A. Grimaud, and F. Tournemaine, 2012. "Pricing knowledge and funding research of new technology sectors in a growth model". Journal of Public Economic Theory 14 (3): 493-520.

Corrado, C., C. Hulten, and D. Sichel, 2009. "Intangible capital and U.S. economic growth". Review of Income and Wealth, 55 (3): 661-685.

Cozzi, G. 1998. "Culture as a bubble". Journal of Political Economy 106 (2): 376-394.

Cozzi, G., and L. Spinesi, 2004. "Information transmission and the bounds to growth". Topics in Economic Analysis \& Policy, ISSN (Online) 1538-0653. https://doi.org/10.2202/1538-0653.1168

Cozzi, G. 2017. "Endogenous growth, semi-endogenous growth... or both? A simple hybrid model". Economics Letters 154: 28-30.

Dasgupta, D. 2001. "Lindahl pricing, nonrival infrastructure, and endogenous growth". Journal of Public Economic Theory 3 (4): 413-430.

Eckert, A., and C. Langinier, 2013. "A Survey of the economics of patent systems and procedures". Journal of Economic Surveys 28(5): 996-1015.

Gordon, J. R. 2014. "The demise of U.S. economic growth: restatement, rebuttal and reflections". NBER Working Paper No. 19895.

Gray, E., and A. Grimaud, 2016. "The Lindahl equilibrium in Schumpeterian growth models". Journal of Evolutionary Economics 26 (1): 101-142.

Grossman, G., and E. Helpman, 1991. Innovation and growth in the global economy. Cambridge, MA: MIT Press.

Hellwig, M., and A. Irmen, 2001. "Endogenous technical change in a competitive economy". Journal of Economic Theory, 101 (1): 1-39.

Jones, C. I., 1995. "R\&D-based models of economic growth". Journal of Political Economy 103 (4): 759-784.

Jones, C. I., 1999. "Growth: with or without scale effects?". American Economic Review 89 (2): $139-144$.

Jones, C. I., 2005. "Growth and ideas". In Handbook of economic growth Vol. 1B, edited by P. Aghion and S. Durlauf, 1063-1111. Amsterdam: Elsevier.

Karabarbounis, L., and B. Neiman, 2014. "The global decline of the labor share". Quarterly Journal of Economics 129 (1): 61-103.

Koh, D., R. Santaeulàlia-Llopis, Y. Zheng, 2016. "Labor share decline and intellectual property products capital". SSRN Working Paper. http://dx.doi.org/10.2139/ssrn.2546974

Marchese, C., S. Marsiglio, F. Privileggi, and G. B. Ramello, 2017. "Endogenous recombinant growth through market production of knowledge and intellectual property rights". Macroeconomic Dynamics: $1-33$.

https://doi.org/10.1017/S136510051700058X

Marchese, C., and F. Privileggi, 2016. "A competitive idea-based growth model with shrinking 
workers' income share". Dept. "Cognetti de Martiis" Working Paper No. 4/2016: 1-36. http://www.est.unito.it/do/home.pl/Download?doc=/allegati/wp2016dip/wp_4_2016.pdf

Marchese, C., and F. Privileggi, 2018. "Endogenous economic growth with disembodied knowledge". Journal of Public Economic Theory 20 (3): 437-449. https://doi.org/10.1111/jpet.12284

Peretto, P. F. (1996). "Sunk costs, market structure, and growth". International Economic Review 37 (4): 895-923.

Peretto, P. F. 1998. "Technological change and population growth". Journal of Economic Growth 3 (4): 283-311.

Romer, P. M. 1990. "Endogenous technological change". Journal of Political Economy 98 (5): S71-S102.

Schumpeter, J. A. 1911. The Theory of Economic Development. Cambridge, MA: Harvard University Press.

Scotchmer, S. 2005. Innovation and Incentives. Cambridge, MA: MIT Press.

Tsur, Y., and A. Zemel, 2007. "Towards endogenous recombinant growth". Journal of Economic Dynamics and Control 31 (11): 3459-3477.

Weitzman, M. L. 1998. "Recombinant growth". The Quarterly Journal of Economics 113 (2): $331-360$. 